\title{
VIRTUAL LABORATORIES IN EDUCATION
}

A cheap way for schools to obtain laboratories for all courses, by using the Computer Laboratoryt

\author{
Antonios Alexiou, Christos Bouras and Eleftheria Giannaka \\ Research Academic Computer Technology Institute, Greece and Computer Engineering and \\ Informatics Dept., Univ. of Patras, Greece
}

\begin{abstract}
Practical experience is an important component of the educational process. However, the time and economical resources often required for the setting up and construction of scientific laboratories is outside the scope of many institutions. A solution to this problem could be found in the adaptation of the Virtual Reality technology, which could allow the creation of Virtual Laboratories, which will simulate the processes and actions that could take place in real laboratories. In particular, this paper, based on the expertise and motivation gained by the VirRAD-IST project, proposes and describes such an educational virtual laboratory, which aims to meet the requirements of a real laboratory and furthermore to support communication and collaboration services. We propose a web-based system, which allows users to perform experiments on educational fields, such as Physics or Chemistry in 3D multiuser worlds where users are represented by avatars and they are offered a wide range of communication and collaboration services in order to simulate efficiently a real learning experimental process.
\end{abstract}

Key words: Distance learning, Virtual reality, Multi-user virtual environments, Virtual Laboratories, Educational Virtual Environments.

\section{INTRODUCTION}

The wide expansion of the World Wide Web (WWW) and the maturation of the Internet along with the step-up of both the network infrastructure and

1 The work presented in this paper is supported by VirRAD European project, Project No: IST-2001-32391 
the electronic communication formed all the necessary preconditions for adopting this powerful means of communication for accessional purposes.

Regarding the technological field, Virtual Reality (VR) technology has been widely proposed and recognized as a major technological advance for supporting life-long education to individuals along with a flexible workforce. One of the unique capabilities of the VR technology is the successful translation of abstract concepts into visualized events along with the possibility of the users' interaction with them, that in real life could be limited due to distance, time and safety factors ${ }^{1}$.

The extended functionalities that a Virtual Environment could provide, in combination to the increasing research interest for distance learning led to the creation of a wide range of applications that adopt Virtual Reality technology in order to support the learning process and such forming the so called Educational Virtual Environments. However, apart from the technological aspects that Virtual Reality embraces, the term "educational" posts a number of issues, e.g. on the access of the learning content as well as on the educational model that should be used for the optimized realization of the learning process. One of the most evolving trends to this direction is experimental learning or "learning by experience" where users are encountered as actors and not as passive receivers of the information provided. Experimental learning involves a high degree of interactivity of the users both with other users as well as with the objects available in the Virtual Environment ${ }^{3}$. A case of such an environment is the Virtual Radiopharmacy Laboratory, created on the framework of the VirRAD IST project, which will be presented in a following section. Furthermore, another example of a distributed virtual reality environment for simulation-based learning is C-VISions. C-VISions is a research project, which focuses on the implementation of a multi-user 3D environment for educational purposes. In order the learning process to be realized, users are represented by avatars in a $3 \mathrm{D}$ environment 1 .

The evolution noticed in Information and Communication Technologies has also affected Greece, both in the industrial as well as in the educational field. In regard to the educational field, it should be mentioned that the last decade the majority of secondary schools have been equipped with computer laboratories in order the students to become familiar with the information society and the new technologies. In addition, a network for the internal connection of all schools has been established, allowing the communication and collaboration of individuals in the field of secondary education. However, the educational system has not taken full advantage of additional capabilities that the school computer laboratories could present. Therefore, this paper emphasizes on the description of a platform that Greek Secondary 
Schools could adopt in order trainees to meet "Learning by experience" and collaboration, over the Greek School Network" ${ }^{5}$.

The remainder of this paper is structured as follows. In section 2 fundamental issues of Virtual Laboratories are apposed and their role in education is underlined. In section 3, a Virtual Laboratory called Virtual Radiopharmacy Laboratory is presented. Section 4 is dedicated to a proposed scheme that could allow the creation of Virtual Laboratories in Greek secondary schools. Following this, the functionality provided by these Virtual Laboratories is presented. Finally, some concluding remarks are briefly described.

\section{VIRTUAL LABORATORIES IN EDUCATION}

Practical experience is an important component of the educational process. However, the time and economical resources required for the setting up and construction of scientific laboratories is outside the scope of many institutions. A solution to the above problem could be found in the adaptation of Virtual Laboratories, which could be proven as an important educational tool that deals with the lack of practical experience in education ${ }^{2}$.

Among the many benefits of virtual laboratories, the following are particularly important:

1. Resource sharing becomes a reality, improving the utilization of costly equipment.

2. Access to educational and research material is facilitated for both students and professionals.

3. Scientific investigation standards are established in areas where practical experimentation is a required part of research.

4. Reduction in travel time leads to productivity enhancements

A virtual laboratory that uses VR technology to support e-learning is the Virtual Radiopharmacy Laboratory which is described in the following section.

\subsection{The Virtual Radiopharmacy Laboratory}

The Virtual Radiopharmacy Laboratory is a 3D simulation of a real radiopharmacy laboratory where learners, represented by 3D avatars, can experiment on radiopharmacy equipment by carrying out specific learning scenarios. This laboratory has been implemented in the framework of the VirRAD European project, which has as main goal to support the Radiopharmacists' community worldwide. 
One of the most powerful characteristics of the Virtual Radiopharmacy Laboratory is that it aims to support both RBL and collaborative e-learning, by being accessed in two different modes: the study and the multi-user mode.

In the study mode, as it is shown in Fig. 1, the user can interact with the environment without the presence of other users. His/her help is an intelligent learner modeling that provides assistance to the user according to the dynamic actions that $\mathrm{s} / \mathrm{he}$ performs on the available equipment of the virtual space.

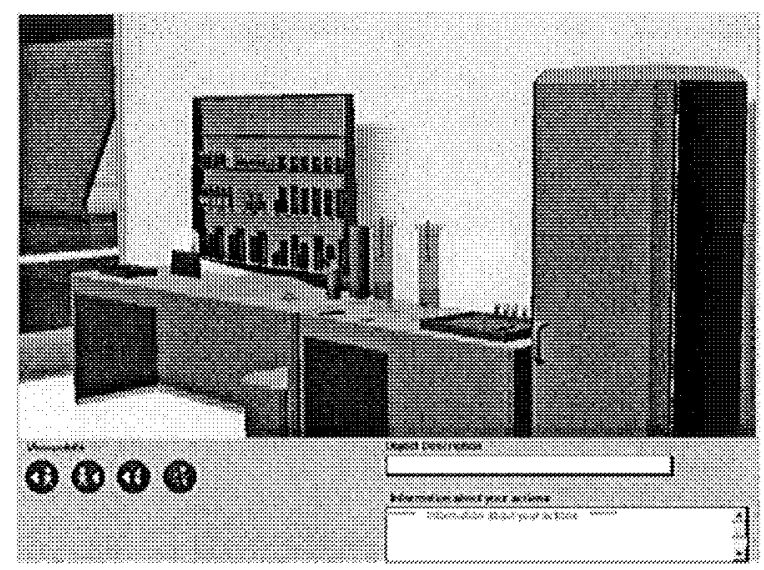

Figure 1. Virtual Radiopharmacy Laboratory: The study mode

The multi-user mode of the Virtual Radiopharmacy Laboratory, as it is shown in Fig. 2, provides a virtual environment that allows learners to interact with other learners / mentors or with the equipment of the laboratory, exactly as it would happen in a real radiopharmacy laboratory.

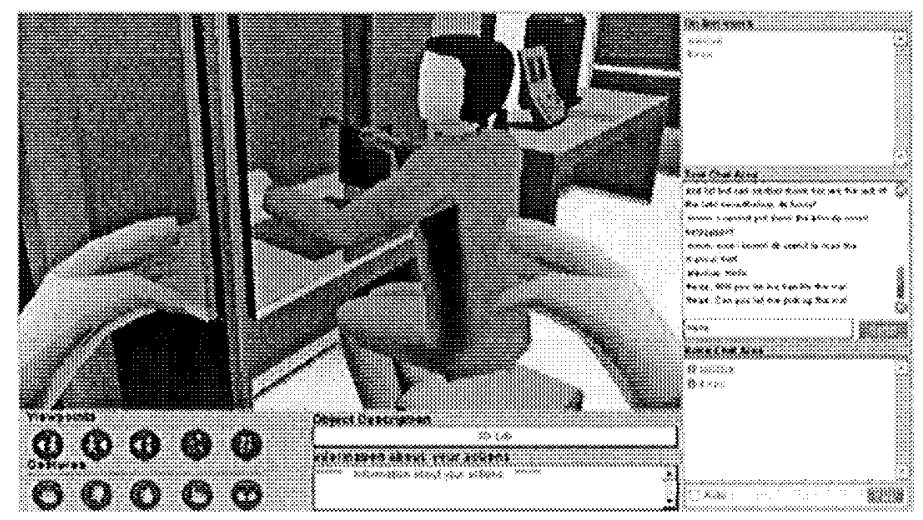

Figure 2. Virtual Radiopharmacy Laboratory: The multi-user mode. 
Thus, through his/her 3D representation, every participant has the ability to communicate, discuss and cooperate with other participants and as a team perform actions in the laboratory, having in mind that these actions must be in accordance with the specific learning scenario ${ }^{1}$.

\section{VIRTUAL LABORATORIES IN GREEK SECONDARY SCHOOLS}

The last years the majority of Greek secondary schools have been equipped with computer laboratories so that learners can become familiar with the information society and the new technologies that arise. In addition a network, the Greek School Network, has been established for connecting the secondary schools to the Internet and for providing educational network services. In particular, the computer laboratories are mainly used for the tuition of the Informatics course, where learners are introduced to some primary programming and algorithms as well as to the use of the Internet ${ }^{5}$.

However, the educational system has not taken full advantage of additional enhanced abilities that the school computer laboratories and the new technologies could provide. Therefore, the approach of this paper is that by combining the school computer laboratory, the experience that the students gain from the teaching of the information science course and the powerful capabilities of Virtual Reality technology there could be developed Virtual Laboratories for various lessons such as Physics and Chemistry.

The benefits that the adaptation of Networked Virtual Environments could present for the learning process plead to this direction. In particular, a networked virtual environment could sub serve the learning process in two ways:

1. Creation of Virtual Courses: Each trainee is represented by a 3D entity with human-like characteristics and through this representation s/he can collaborate with the other trainees as well as attend the tutor during the session of the Virtual Course.

2. Simulation of school laboratories: In many cases the creation of real laboratories for special kind of courses is limited due to practical or economical constraints.

The Virtual Laboratories proposed in this paper involve the design, development and realization of pre-determined experiments that will simulate phenomena and processes that could take place in real-world situations. The above-mentioned laboratories could act either as complement of the existing school laboratories or as independent laboratories, especially for courses where the real laboratories cannot be developed for practical reasons. 
The anticipated Virtual Laboratories have as main target to form Educational Virtual Environments in order to provide to the Greek secondary schools additional practical educational material and experiences in fields of tuition, such as Physics. In this case and in order to achieve the above goal the Virtual Laboratory designed will simulate a set of Physics experiments that could take place in a real Physics laboratory along with special experiments that could not be easily executed in real laboratories.

Finally, one of the most important elements of this proposal is the scalability that the approach maintains through the adaptation of open source technologies. In particular, both the platform that supports the Virtual Environments and the simulators of the experiments can be developed with open source technologies, as VRML. Furthermore, the final platform that will allow the creation and will support the Virtual Laboratories can be provided as open source application, free of charges and limitations that are commonly related to proprietary technologies.

\subsection{Proposed Architecture}

This section is dedicated to the description of the proposed architecture of the system that will support the Virtual Laboratories in Greek Secondary Schools. The architecture refers to the logical view of the static structure in terms of its components, their interconnections, as well as the interfaces and operations offered by these components.

The system architecture, which is presented in Fig. 3, is based on the following components for providing the needed functionality: a database, the platform that supports multi-user environments and simulations, a web server and the Greek School Network which connects the system with the participants' clients. These components are described below in detail.

The database of the system stores the information about students such as their name and password, their profiles, their role in the system that could be either tutor or learner, as well as the avatar they have selected for their representation in the Virtual Environment.

The Networked Virtual Environment (NVE) server, which is the multiuser platform, provides and maintains a consistent view of the Virtual Laboratories and of the shared objects in it. In addition it provides communication and collaboration tools such as text and audio chat, application sharing, etc in order to simulate as more realistic as possible the interactions of the participants.

As it is depicted in Fig. 3 the users can access the system using a web browser. The web server can be used in order to host the 3D training areas where simulations take place, the client-side files of the Virtual Laboratories and the avatars of the users. Furthermore, the web server stores and executes 
the scripts in order to obtain and manipulate the users' data from the database.

The connection of the client's browser and the platform is materialized over the Greek School Network, which is an advanced educational network that connects all school computer laboratories and creates an educational intranet. This national infrastructure provides and supports advanced telematics services to the school communities. The design model and the operational specifications of the GSN are based on the TCP/IP protocol.

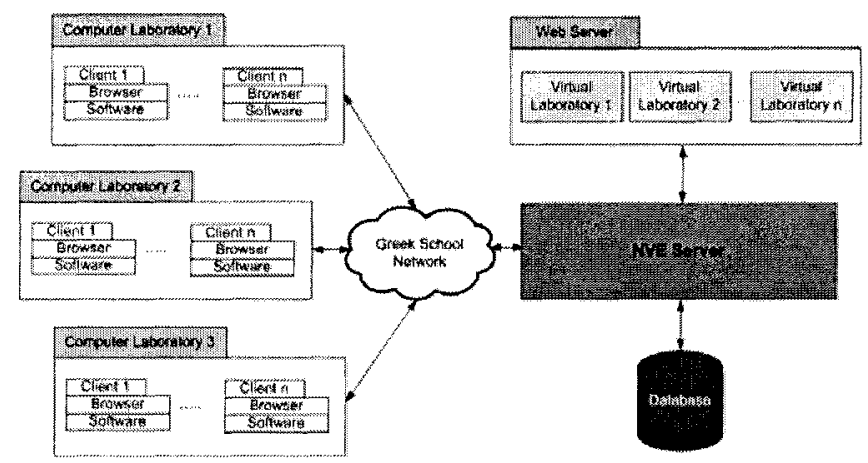

Figure 3. Proposed Architecture

As mentioned above, the systems architecture constitutes a simple structure, which, however, could allow and support efficiently the basic functionality that the platform is intended to offer.

\subsection{Basic Functionality}

The main goal of an Educational Virtual Laboratory is to provide all the simulations, tools, applications and conditions necessary, which will constitute an efficient space where experimentation, communication and collaboration can be used for the maintenance and exchange of rich knowledge $e^{3}$. Thus, the virtual environment that will host the laboratories, trying to simulate the learning process from its very beginning until its completion, should, first of all, include all the necessary functionality to its users so as to simulate the real processes as more realistic as possible. The functionality diagram of a Virtual Laboratory is displayed in Fig. 4.

The Virtual Laboratories comprise Virtual Worlds where users are represented by $3 \mathrm{D}$ human like avatars and have the ability to navigate in the environment, conduct experiments by interacting with the simulated equipment and collaborate by interacting and communicating with the other participants. Therefore, in a Virtual Laboratory where Physics is selected, for example as the application field, tutors will be provided with the ability to 
teach the each time selected course while the learners will have the ability to perform experiments in fields such as electricity, magnetism, mechanics and other fields of Physics without the necessary existence of conventional accoutrements.

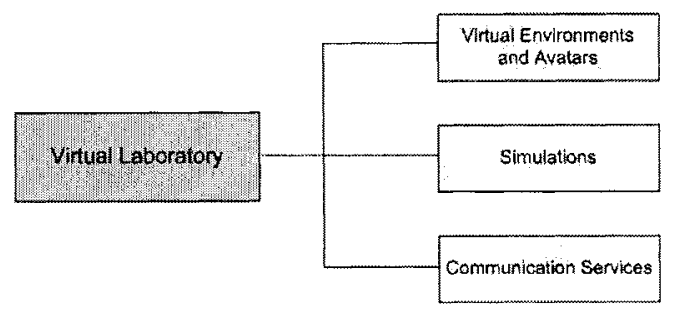

Figure 4. Functionality diagram of a Virtual Laboratory

For the achievement of the purpose that a platform like this one could have, the functionality that will be provided by the Virtual Laboratory can vary in respect to the kind of the laboratory that will be simulated ${ }^{4}$. However, in all cases the platform should support the following:

a) Creation of Virtual Laboratories, where the placement and manipulation of objects that will be used to the experiments will be possible.

b) Representation of the users, both tutors and learners through human like entities, called avatars.

c) Creation of dynamic characteristics and physical attributes to the objects as gravity, reflection, etc.

d) Definition of the possible ways of communication.

The aforementioned functionality can supply a wide range of the requirements that the learning process could have in a real laboratory. Such an environment could support learning by experience even in cases that either the lack of resources for the creation of real laboratories or the deficiency of available spaces constrain the learning process.

The benefits of such an approach are the following:

1. Significantly reduced cost for the composition of a school laboratory, as it only requires addition of the necessary software to the existing Informatics Laboratory.

2. Mistakes of both tutors and learners cannot be proven catastrophic for both the lab and the participants' health.

3. The interaction with the virtual environment motives the users, especially the learners more in regard to the real process.

4. This idea allows the simulation of Laboratories that could only exist in computers and could not be realized in real conditions.

As extracted from the above, the first criterion for the design and the development of a virtual laboratory is the selection of the field that will be 
adopted for the simulation processes. This field will define the procedures that should be simulated as well as the learning process that will lead to the achievement of knowledge and experience. The next step is to provide to the end users all necessary services so as to reach a high sense of realism.

\section{CONCLUSIONS}

As the Internet is turning into a truly multiservice network with a steady increase in bandwidth and decrease in response time, the environment becomes more suitable for implementations such as virtual laboratories using VR technology.

Traditionally, the laboratory has been an integral component of education for relating fundamental concepts to basic real-world phenomena. Conducted largely within the confines of the lecture-classroom format, the laboratory involves the use of largely pre-determined experiments that simulate basic phenomena found in real-world situations. Unfortunately, the lectureclassroom-laboratory system has its flaws or demanding requirements: timespace dislocations of what is taught, what is explained and learned, and what is practiced and internalized, as well as the need for large amounts of space and complicated logistics to create and maintain an appropriate learning environment.

However, the role of conventional laboratories for training and understanding educational problems is presently being challenged. The advances made by Information Technology has led to some learning processes being replicated to varying degrees in the form of courseware available through the Internet, thereby overcoming some limitations of the traditional lecture-classroom-laboratory system.

Virtual laboratories seem to be preferable to real laboratories in the following cases:

1. The learning procedure through real laboratories puts students life in jeopardy.

2. Some experiments cannot be performed due to time constrains or hazards.

3. The cost in order to construct or to preserve a real laboratory is forbidden.

4. There are experiments that can be simulated only in computers.

Virtual Reality provides a friendly representation of the provided information, interaction with the system, which does not require advanced knowledge of the computers technology and reduced cost compared to other technologies. In addition, one of the most important aspects of this proposal is the fact that the development of Virtual Laboratories can be fully 
supported by the existing Computer Laboratories, without the need of additional investments and infrastructure.

\section{FUTURE WORK}

The step that follows the design of the system is to proceed with the implementation of the Virtual Laboratory. Firstly, there should be a decision on the educational field that is going to be simulated by the virtual laboratory, for example the Physics or Chemistry Laboratory.

When this field is defined, the equipment and the actions that will be simulated should be defined so as the models and the simulations to be designed and implemented respectively.

Following the gathering of the necessary information is the actual implementation of the multi-user platform that will support the simulations as well as the communication and collaboration services.

The next step is to conduct a user evaluation by setting up the platform in two selected Secondary Schools so as to get feedback on the correctness of the scenarios simulated as well as on the usability and friendliness of the represented information.

Finally, the platform will be provided to all Greek Secondary Schools as Open Source application, which will run over the already existing Greek School Network.

\section{REFERENCES}

1. A. Alexiou, C. Bouras, E. Giannaka, V. Kapoulas, M. Nani, T. Tsiatsos, Using VR technology to Support e - Learning: The 3D Virtual Radiopharmacy Laboratory, 6th International Workshop on Multimedia Network Systems and Applications, Tokyo, Japan, March 2004, pp. 268-273

2. E. Guimaraes, A. Maffeis, J. Pereire, B. Russo, E. Cardoso, M. Bergerman, M. Magalhaes, REAL: A virtual laboratory for mobile robot experiments, IEEE Trans. Educ., vol. 46, , Feb. 2003, pp. 37-42.

3. M. Kubo, R. Tori, C, Kimer, Interaction in collaborative educational virtual environments, Journal in Cyberpsychology Behavior, Oct. 2002, pp. 399-407.

4. C. Bouras, V. Triantafillou, T. Tsiatsos, A Framework for Intelligent Virtual Training Environment: The steps from specification to design, Journal of International Forum of Educational Technology \& Society (IFETS) Special Issue on Innovations in Learning Technology, Learning Technology Task Force - IEEE (on - line), Vol. 5, Issue 4, 2002, pp. 11-26

5. C. Bouras, M. Paraskeuas, Educational Information Society in Greece: The Greek School Network, IADIS International Conference e - SOCIETY, Lisbon, Portugal, June 2003, pp. $179-186$. 